\title{
THE INNER DERIVATIONS OF A JORDAN ALGEBRA
}

\author{
BY JOHN R. FAULKNER ${ }^{1}$
}

Communicated by A. D. Mostow, September 21, 1966

A Jordan algebra $J$ is an algebra over a field $\Phi$ of characteristic $\not \neq 2$ with a product $a \cdot b$ satisfying

$$
\begin{aligned}
a \cdot b & =b \cdot a, \\
\left(a^{\cdot 2} \cdot b\right) \cdot a & =a^{\cdot 2} \cdot(b \cdot a)
\end{aligned}
$$

where $a^{\cdot 2}=a \cdot a$. The following operator identity is easily derived from (1) and the linearized form of (2)

$$
\left[a_{R}\left[b_{R} c_{R}\right]\right]=\left(a\left[b_{R} c_{R}\right]\right)_{R} \quad \text { for } a, b, c, \in J
$$

where $x_{R}$ denotes right multiplication by $x$ and $[u v]=u v-v u$. Letting $D=\left[b_{R} c_{R}\right]$, we see that (3) implies $(d \cdot a) D-(d D) \cdot a=d \cdot(a D)$ for $a, d \in J$. In other words, $D$ is a derivation of the Jordan algebra $J$. Hence every mapping of the form $\sum\left[b_{i R} c_{i R}\right]$ is a derivation. We shall call such derivations inner derivations and denote the set of all inner derivations of $J$ by $\operatorname{Inder}(J)$. It is easily shown that $\operatorname{Inder}(J)$ is an ideal in the Lie algebra of all derivations of $J$. We shall show that if the characteristic of $\Phi$ is $p \neq 0$, then $\operatorname{Inder}(J)$ is a restricted Lie algebra; that is, $D^{p} \in \operatorname{Inder}(J)$ if $D \in \operatorname{Inder}(J)$.

If $\mathfrak{A}$ is an associative algebra, we denote by $\mathfrak{A}^{+}$the Jordan algebra whose vector space is that of $\mathfrak{A}$ and whose multiplication is $u \cdot v$ $=\frac{1}{2}(u v+v u)$. A Jordan algebra $J$ is special if $J$ is a subalgebra of $\mathfrak{Q}^{+}$ for some associative algebra $\mathfrak{A}$. Let $\Phi\left\{x_{1}, \cdots, x_{n}\right\}$ be the free associative algebra generated by $x_{1}, \cdots, x_{n}$ over the field $\Phi$. An element $u$ in $\Phi\left\{x_{1}, \cdots, x_{n}\right\}$ is called Jordan if $u$ is in the subalgebra of $\Phi\left\{x_{1}, \cdots, x_{n}\right\}+$ generated by 1 and $x_{1}, \cdots, x_{n}$. We can now state the following

Lemma. If $\Phi$ is of characteristic $p \neq 0,2$, then there exist Jordan elements $f_{i}(x, y), i=1,2$ in $\Phi\{x, y\}$ such that $[x y]^{p}=\left[x, f_{1}(x, y)\right]$ $+\left[y, f_{2}(x, y)\right]$.

Proor. We introduce the reversal operation in $\Phi\{x, y\}$ which is an involution $a \rightarrow a^{*}$ such that $x^{*}=x$ and $y^{*}=y$. We say $a$ is reversible if $a^{*}=a$. Let $\mathfrak{M}$ be the subspace of $\Phi\{x, y\}$ of all elements of the form $[x a]+[y b]$ where $a$ and $b$ are reversible. Since by Cohn's theorem

1 The author is a National Science Foundation graduate fellow. The author also wishes to thank Professor N. Jacobson, who originally suggested this research. 
[1] every reversible element of $\Phi\{x, y\}$ is a Jordan element, we need only show that $[x y]^{p} \in \mathfrak{M}$.

Let $A$ be the set consisting of the $2^{p}$ monomials of the form $u=a_{1} a_{2}$ $\cdots a_{p}$ where $a_{i}=x y$ or $-y x, i=1,2, \cdots, p$. We define an equivalence relation $\sim$ on $A$ by $u \sim v$ if $v=a_{1 \sigma} a_{2 \sigma} \cdots a_{p \sigma}$ where $u$ is as above and $\sigma$ is a cyclic permutation of $(1,2, \cdots, p)$. An equivalence class determined by $\sim$ has either 1 or $p$ elements since the cyclic permutations of $(1,2, \cdots, p)$ form a cyclic group of order $p$. Let $A_{1}=\left\{u_{11}=(x y)^{p}\right\}, A_{2}=\left\{u_{21}=(-y x)^{p}\right\}, A_{3}=\left\{u_{31}, u_{32}, \cdots, u_{3 p}\right\}$, $\cdots, A_{c}=\left\{u_{e 1}, u_{e 2}, \cdots, u_{e p}\right\}$ be the equivalence classes determined by $\sim$.

If $r=b_{1} b_{2} b_{3} \cdots b_{2 p}$ and $s=b_{2} b_{3} \cdots b_{2 p} b_{1}$ where $b_{i}=x$ or $y, i=1,2,3$, $\cdots, 2 p$, then $\left(r-r^{*}\right)-\left(s-s^{*}\right)=\left[b_{1}, b_{2} b_{3} \cdots b_{2 p}+b_{2 p} \cdots b_{3} b_{2}\right] \in \mathfrak{M}$. Thus, if $t=b_{1 r} b_{2 \tau} \cdots b_{(2 p) \tau}$ where $\tau$ is a cyclic permutation of $(1,2, \cdots, 2 p)$, then $\left(r-r^{*}\right)-\left(t-t^{*}\right) \in \mathfrak{M}$. In particular, $\left(u-u^{*}\right)$ $-\left(v-v^{*}\right) \in \mathfrak{M}$ if $u, v \in A$ and $u \sim v$. Also, $\left(u_{11}-u_{11}{ }^{*}\right)+\left(u_{21}-u_{21}{ }^{*}\right) \in \mathfrak{M}$.

Now we may write $[x y]^{p}=(x y-y x)^{p}=\sum u \in A u$. Since $\left([x y]^{p}\right)^{*}$ $=-[x y]^{p}$, we have

$$
\begin{aligned}
{[x y]^{p} } & =\frac{1}{2} \sum u \in A\left(u-u^{*}\right) \\
& =\frac{1}{2}\left\{u_{11}-u_{11}^{*}+u_{21}-u_{21}^{*}+\sum_{i=3}^{\dot{j}} \sum_{j=1}^{p}\left(u_{i j}-u_{i j}^{*}\right)\right\} \\
& =\frac{1}{2}\left\{m+\sum_{i=3}^{e}\left(p\left(u_{i 1}-u_{i 1}^{*}\right)+m_{i}\right)\right\}
\end{aligned}
$$

where $m, m_{i} \in \mathfrak{M}, i=3, \cdots, p$. Hence $[x y]^{p} \in \mathfrak{M}$.

THEOREM. If the characteristic of $\Phi$ is $p \neq 0$, then the Lie algebra Inder $(J)$ is restricted.

Proor. We recall the following two identities which hold in any associative algebra over $\Phi[2$, pp. 186-187]:

$$
\begin{gathered}
u(\operatorname{ad} v)^{p}=u\left(\operatorname{ad} v^{p}\right), \\
(u+v)^{p}=u^{p}+v^{p}+\sum_{i=1}^{p-1} s_{i}(u, v)
\end{gathered}
$$

where $x(\operatorname{ad} y)=[x y]$ and $s_{i}(u, v)$ is in the Lie subalgebra generated by $u$ and $v$. Let $D=\sum\left[b_{i R} c_{i R}\right] \in \operatorname{Inder}(J)$. In view of (5), we will have $D^{p} \in \operatorname{Inder}(J)$ if $\left[b_{R} c_{R}\right]^{p} \in \operatorname{Inder}(J)$ for $b, c \in J$.

First we assume that $J$ is special. By writing both sides in terms of the associative multiplication, one verifies the following identity 


$$
a\left[b_{R} c_{R}\right]=\left(\frac{1}{4}\right)[a[b c]] \quad a, b, c \in J .
$$

As an immediate consequence of (6) and (4) we have

$$
a\left[b_{R} c_{R}\right]^{p}=\left(\frac{1}{4}\right)^{p}\left[a[b c]^{p}\right] \quad a, b, c \in J .
$$

Using the lemma, we may write

$$
[b c]^{p}=\left[b f_{1}(b, c)\right]+\left[c f_{2}(b, c)\right] \quad b, c \in J .
$$

Combining (7) and (8) and making use of (6), we see

$$
\text { (9) } a\left[b_{R} c_{R}\right]^{p}=a\left(\frac{1}{4}\right)^{p-1}\left\{\left[b_{R}\left(f_{1}(b, c)\right)_{R}\right]+\left[c_{R}\left(f_{2}(b, c)\right)_{R}\right]\right\} \quad a, b, c \in J \text {. }
$$

Since (9) involves only $a, b$, and $c$ with $a$ linear and since (9) holds for all special Jordan algebras over $\Phi$, it must hold for all Jordan algebras over $\Phi$ by MacDonald's theorem [3]. Thus $\left[b_{R} c_{R}\right]^{p}$ $\in \operatorname{Inder}(J)$, and $\operatorname{Inder}(J)$ is restricted.

\section{REFERENCES}

1. P. M. Cohn, On homomorphic images of special Jordan algebras, Canad. J. Math. 6 (1954), 253-264.

2. Nathan Jacobson, Lie algebras, Interscience, New York, 1962.

3. I. G. MacDonald, Jordan algebras with three generators, Proc. London Math. Soc. 10 (1960), 395-408.

YALE UNIVERSITY 